\title{
Proof of Two Conjectures of Brenti and Simion on Kazhdan-Lusztig Polynomials
}

FABRIZIO CASELLI

caselli@igd.univ-lyon1.fr; caselli@mat.uniroma1.it Dipartimento di Matematica “G. Castelnuovo”, Università di Roma “La Sapienza”, P. le A. Moro 5, 00185, Roma, Italy

Received April 25, 2002; Revised December 26, 2002; Accepted January 14, 2003

Abstract. We find an explicit formula for the Kazhdan-Lusztig polynomials $P_{u_{i, a}, v_{i}}$ of the symmetric group $\mathfrak{S}(n)$ where, for $a, i, n \in \mathbb{N}$ such that $1 \leq a \leq i \leq n$, we denote by $u_{i, a}=s_{a} s_{a+1} \cdots s_{i-1}$ and by $v_{i}$ the element of $\mathfrak{S}(n)$ obtained by inserting $n$ in position $i$ in any permutation of $\mathfrak{S}(n-1)$ allowed to rise only in the first and in the last place. Our result implies, in particular, the validity of two conjectures of Brenti and Simion [4, Conjectures 4.2 and 4.3], and includes as a special case a result of Shapiro, Shapiro and Vainshtein [13, Theorem 1]. All the proofs are purely combinatorial and make no use of the geometry of the corresponding Schubert varieties.

Keywords: Kazhdan-Lusztig polynomials, symmetric group, Bruhat order

\section{Introduction}

In [7] Kazhdan and Lusztig defined, for every Coxeter system $W$, a family of polynomials, parametrized by pairs of elements of $W$, which have become known as the Kazhdan-Lusztig polynomials of $W$. These polynomials are intimately related to the Bruhat order of $W$ and have proven to be of fundamental importance in representation theory and in the geometry of Schubert varieties. We will focus our attention to the case of the symmetric group. Despite the rather elementary recursion relations they satisfy, these polynomials are in general quite difficult to compute. In fact the only families of Kazhdan-Lusztig polynomials that are known explicitly correspond to situations where the geometry of the corresponding Schubert varieties is easier (see, for example, $[1,10,12]$ and [13, Theorems 1 and 2]), where the interval $[u, v]$ has some special property (see, for example, [2, Corollaries 6.8 and 6.9]) or when the shape of the indexing permutations lead in some natural way to the use of induction (see, for example, [4, Corollary 3.2 and Theorem 3.3] or [11]). This work gives results in the direction of explicit formulae for the Kazhdan-Lusztig polynomials of the symmetric group when the indexing permutations are of particular forms.

The main results are the following. First we reduce the calculation of $P_{u, v}(q)$ when $u, v \in \mathfrak{S}(n)$ satisfy $u^{-1}(n)-v^{-1}(n) \leq 3$ to an (easier) problem in $\mathfrak{S}(n-1)$. Then we focus our attention on permutations in $\mathfrak{S}(n)$ that are obtained from an element of $\mathfrak{S}(n-1)$ allowed to rise only in the first and in the last position by inserting $n$ (or 1) anywhere in its complete notation. We write down certain recurrence relations satisfied by some related KazhdanLusztig polynomials and we obtain explicit formulae from these relations. Finally, as an 
application of this result, we find explicit formulae for $P_{\boldsymbol{e}, \sigma(n-2) \sigma(n-1) \sigma(n) n-3 \cdots 4 \tau(1) \tau(2) \tau(3)}$ where $(\sigma, \tau) \in \mathfrak{S}(3) \times \mathfrak{S}(3) \backslash(e, e)$ act on the set $\{n-2, n-1, n, 1,2,3\}$ in the most natural way, establishing, in particular, two conjectures due to F. Brenti and R. Simion (see [4, Conjectures 4.2 and 4.3]). The proofs rely on the special shape of the permutations under consideration that will allow us to deduce some easy recursions satisfied by these polynomials with no use of geometry.

\section{Notation and preliminaries}

In this section we collect some definitions and results that are used in the proofs of this work.

We let $\mathbb{N}:=\{0,1,2,3, \ldots\}$ be the set of non-negative integers and for $a \in \mathbb{N}$ we let $[a]:=\{1,2, \ldots, a\}$ (where $[0]=\emptyset$ ). For $b \in \mathbb{R}$ we let $\lfloor b\rfloor$ be the largest integer $\leq b$. Given $n, m \in \mathbb{N}, n \leq m$, we let $[n, m]:=\{n, n+1, \ldots, m\}$. We write $S=\left\{a_{1}, \ldots, a_{r}\right\}_{<}$to mean that $S=\left\{a_{1}, \ldots, a_{r}\right\}$ and $a_{1}<\cdots<a_{r}$. For a sequence $i_{1}, i_{2}, \ldots, i_{n}$ and $j \in[n]$, we denote by $i_{1}, \ldots, \widehat{i_{j}}, \ldots, i_{n}$ the subsequence $i_{1}, \ldots, i_{j-1}, i_{j+1}, \ldots, i_{n}$ obtained by suppressing the entry $i_{j}$.

For $i \in \mathbb{Z}$ we denote by

$$
[i]_{q}:=\sum_{j=0}^{i-1} q^{j}
$$

so that $[i]_{q}=0$ if $i \leq 0$. Given a polynomial $P(q)$ and $i \in \mathbb{N}$ we denote by $\left[q^{i}\right](P(q))$ the coefficient of $q^{i}$ in $P(q)$.

Given a set $T$ we let $\mathfrak{S}(T)$ be the set of all bijections of $T$. To simplify the notation we denote by $\mathfrak{S}(n)$ instead of $\mathfrak{S}([n])$ the symmetric group on $n$ elements and we denote by $e$ the identity of $\mathfrak{S}(n)$. If $\sigma \in \mathfrak{S}([n, n+k])$ for some $n, k \in \mathbb{N}$, then we write $\sigma=\sigma_{1} \sigma_{2} \ldots \sigma_{k+1}$ to mean that $\sigma(n+i)=\sigma_{i+1}$ for $i=0, \ldots, k$, and call this the complete notation of $\sigma$, while we denote by $s_{i}$ the transposition $(i, i+1)$. Given $\sigma, \tau \in \mathfrak{S}(T)$, we let $\sigma \tau:=\sigma \circ \tau$, i.e. we compose permutations as functions, from right to left.

Given $\sigma \in \mathfrak{S}(n)$, the right descent set of $\sigma$ is

$$
D_{R}(\sigma):=\left\{i \in[n-1]: \sigma_{i}>\sigma_{i+1}\right\}
$$

the left descent set is

$$
D_{L}(\sigma):=\left\{i \in[n-1]:\left(\sigma^{-1}\right)_{i}>\left(\sigma^{-1}\right)_{i+1}\right\}
$$

and the length of $\sigma$ is defined by the number of inversions:

$$
\ell(\sigma):=\operatorname{inv}(\sigma):=\#\left\{(a, b) \in[n] \times[n]: a<b, \sigma_{a}>\sigma_{b}\right\}
$$

For example, if $\sigma=635241$ then $D_{R}(\sigma)=\{1,3,5\}, D_{L}(\sigma)=\{1,2,4,5\}$ and $\ell(\sigma)=$ 12. If $u, v \in \mathfrak{S}(n)$ we also denote $\ell(u, v):=\ell(v)-\ell(u)$. 
Throughout this work we view $\mathfrak{S}(n)$ as a poset ordered by the strong Bruhat order. We are not going to define this order in the usual way (see [6, Section 5.9] for its definition), but we shall use the following characterization of it that is due to Ehresmann [5]. For $\sigma \in \mathfrak{S}(n)$ and $j \in[n]$, let

$$
\left\{\sigma^{j, 1}, \ldots, \sigma^{j, j}\right\}_{<}:=\left\{\sigma_{1}, \ldots, \sigma_{j}\right\} .
$$

Theorem 2.1 Let $\sigma, \tau \in \mathfrak{S}(n)$. Then $\sigma \leq \tau$ if and only if $\sigma^{j, i} \leq \tau^{j, i}$ for all $1 \leq i \leq j \leq$ $n-1$.

For $u, v \in \mathfrak{S}(n)$ we also write $u \triangleleft v$ to mean that $u \leq v$ and $\ell(u, v)=1$.

We take the following fundamental result (see [6, Section 7.11] for a proof) as the definition of the Kazhdan-Lusztig polynomials:

Theorem 2.2 (Kazhdan-Lusztig) There exists a unique family of polynomials $\left\{P_{u, v}(q), u\right.$, $v \in \mathfrak{S}(n)\} \subset \mathbb{Z}[q]$ such that:

(1) $P_{u, v}(q)=0$ if $u \not \leq v$;

(2) $P_{u, v}(q)=1$ if $u=v$;

(3) If $u<v$ then

$$
\operatorname{deg}\left(P_{u, v}\right) \leq \frac{\ell(u, v)-1}{2}
$$

(4) If $u<v$ and $i \in D_{R}(v)$ then

$$
P_{u, v}(q)=q^{1-c} P_{u s_{i}, v s_{i}}(q)+q^{c} P_{u, v s_{i}}(q)-\sum_{\left\{z: i \in D_{R}(z)\right\}} q^{\frac{\ell(z, v)}{2}} \mu\left(z, v s_{i}\right) P_{u, z}(q)
$$

where, for $u, v \in \mathfrak{S}(n)$,

$$
\mu(u, w):= \begin{cases}{\left[q^{\frac{1}{2}(\ell(u, w)-1)}\right]\left(P_{u, w}(q)\right),} & \text { if } u<w \text { and } \ell(u, w) \text { is odd } \\ 0, & \text { otherwise }\end{cases}
$$

$c=1$ if $i \in D_{R}(u)$ and $c=0$ otherwise.

An important consequence of Theorem 2.2 is the following:

Proposition 2.3 Let $u, v \in \mathfrak{S}(n)$ be such that $u<v$ and $i \in D_{R}(v)$. Then

$$
P_{u, v}(q)=P_{u s_{i}, v}(q)
$$

It should be remarked that Theorem 2.2 and Proposition 2.3 can be reformulated in a similar way using left descents instead of right descents. An immediate consequence of Proposition 2.3 is the following: 
Corollary 2.4 Let $z, w \in \mathfrak{S}(n), z \leq w$, be such that $\mu(z, w) \neq 0$ and $\ell(z, w)>1$. Then $D_{R}(z) \supseteq D_{R}(w)$ and $D_{L}(z) \supseteq D_{L}(w)$.

Corollary 2.4 motivates the following notation: for $u, v \in \mathfrak{S}(n)$ and $i \in[n-1]$ we let

$$
\begin{aligned}
& Z_{1}(u, v ; i):=\left\{z \in \mathfrak{S}(n): u \leq z \leq v, z \not v, D_{R}(z) \supseteq D_{R}(v) \cup\{i\} \text { and } D_{L}(z) \supseteq D_{L}(v)\right\} \\
& Z_{2}(u, v ; i):=\left\{z \in \mathfrak{S}(n): u \leq z \triangleleft v, i \in D_{R}(z)\right\}
\end{aligned}
$$

and $Z(u, v ; i)=Z_{1}(u, v ; i) \cup Z_{2}(u, v ; i)$ so that Theorem 2.2 can be reformulated in the following way:

Theorem 2.5 Let $u, v \in \mathfrak{S}(n)$ be such that $u \leq v$ and $i \in D_{R}(v)$. Then

$$
P_{u, v}(q)=q^{1-c} P_{u s_{i}, v s_{i}}(q)+q^{c} P_{u, v s_{i}}(q)-\sum_{z \in Z\left(u, v s_{i} ; i\right)} q^{\frac{\ell(z, v)}{2}} \mu\left(z, v s_{i}\right) P_{u, z}(q)
$$

where $c=1$ if $i \in D_{R}(u)$ and $c=0$ otherwise.

Now it is clear that if we want to compute a Kazhdan-Lusztig polynomial using a recursion based on Theorem 2.5 we need to know whenever $\mu\left(z, v s_{i}\right) \neq 0$. This problem is very difficult in general but there are some classes of permutations where it has been solved. Suppose $u, v \in \mathfrak{S}(n)$ are such that $u \leq v \leq(1, n)$. By one of the characterizations of the Bruhat order (see, for example, [6, Section 5.10]) this implies that $u$ and $v$ admit a reduced expression that is a subword of $s_{1} \ldots s_{n-1} \ldots s_{1}$. Fix such expressions and denote them by $\tilde{u}$ and $\tilde{v}$ respectively. Moreover denote by $\tilde{u}_{k}$ and $\tilde{v}_{k}$ the number of occurrences of $s_{k}$ in $\tilde{u}$ and $\tilde{v}$ respectively. For example if $v=4132$ we may choose $\tilde{v}=s_{1} s_{2} s_{3} s_{2}$ so that $\tilde{v}_{1}=1, \tilde{v}_{2}=2$ and $\tilde{v}_{3}=1$. The following result is due to Marietti and its proof can be found in [11, Corollary 6.1]):

Theorem 2.6 Let $u, v \in \mathfrak{S}(n)$ be such that $u \leq v \leq(1, n), \tilde{v}$ and $\tilde{u}$ be two reduced expressions for them that are subwords of $s_{1} \ldots s_{n-1} \ldots s_{1}$. Then $\mu(u, v)=0$ or 1 and it is 1 if and only if there exist $i, j \in \mathbb{N}, 1 \leq i \leq j \leq n-1$ such that

$$
\begin{array}{lll}
\tilde{v}_{k}=\tilde{u}_{k}, & & \text { if } k<i, \\
\tilde{v}_{k}=2 \quad \text { and } \quad \tilde{u}_{k}=1, & \text { if } k=i, \\
\tilde{v}_{k}=2 \quad \text { and } \quad \tilde{u}_{k}=0, & \text { if } i<k \leq j, \\
\tilde{v}_{k}=\tilde{u}_{k}, & & \text { if } k>j .
\end{array}
$$

One more useful property of the function $\mu$ (see [7, Corollary 3.2]) is the following:

Proposition 2.7 Let $u, v \in \mathfrak{S}(n)$. Then

$\mu(u, v)=\mu\left(w_{0} v, w_{0} u\right)$,

where $w_{0}=n \ldots 21$ is the longest element of $\mathfrak{S}(n)$. 
Two other elementary properties of Kazhdan-Lusztig polynomials are the following (see [2, Corollaries 4.3 and 4.4] for proofs):

Proposition 2.8 Let $u, v \in \mathfrak{S}(n)$. Then

$$
\begin{aligned}
P_{u, v}(q) & =P_{u^{-1}, v^{-1}}(q) \\
& =P_{w_{0} u w_{0}, w_{0} v w_{0}}(q) .
\end{aligned}
$$

Let $w \in \mathfrak{S}(n)$. We denote by $\bar{w}$ (respectively $\underline{w}$ ) the permutation of $\mathfrak{S}(n-1)$ obtained from $w$ by suppressing the value $n$ (respectively by suppressing the value 1 and rescaling) from its complete notation. For example, if $w=35214$ then $\bar{w}=3214$ and $\underline{w}=2413$.

Proposition 2.9 Let $u, v \in \mathfrak{S}(n)$ be such that $n$ occurs in the same position in both $u$ and $v$. Then

$$
P_{u, v}(q)=P_{\bar{u}, \bar{v}}(q)
$$

On the other hand, if 1 occurs in the same position in both $u$ and $v$, then

$$
P_{u, v}(q)=P_{\underline{u}, \underline{v}}(q)
$$

Proof: This a special case of [3, Theorem 4.4]. However, the proof of this particular case is so simple that can be included in this work for completeness. We prove only the first statement, the proof of the second being similar. We proceed by induction on $\ell(v)$, the case $\ell(v)=0$ being trivial. Observe that if $u \leq z \leq v$ then $z^{-1}(n)=u^{-1}(n)$ and that if $x, y \in \mathfrak{S}(n), x \leq y$, satisfy $x^{-1}(n)=y^{-1}(n)$, then $\ell(x, y)=\ell(\bar{x}, \bar{y})$. In particular we can suppose that if $\ell(y)<\ell(v)$ then $\mu(x, y)=\mu(\bar{x}, \bar{y})$. If $D_{L}(v) \subseteq\{n-1\}$ then $u \leq v$ implies $u=v$ and the result follows. So suppose $i \in D_{L}(v), i \neq n-1$.

We compute

$$
\begin{aligned}
P_{u, v}(q) & =q^{1-c} P_{s_{i} u, s_{i} v}+q^{c} P_{u, s_{i} v}-\sum_{z: i \in D_{L}(z)} \mu\left(z, s_{i} v\right) P_{u, z} \\
& =q^{1-c} P_{\overline{s_{i} u}, \overline{s_{i} v}}+q^{c} P_{\bar{u}, \overline{s_{i} v}}-\sum_{z: i \in D_{L}(z)} \mu\left(\bar{z}, \overline{s_{i} v}\right) P_{\bar{u}, \bar{z}}
\end{aligned}
$$

by our induction hypothesis and hence

$$
\begin{aligned}
P_{u, v}(q) & =q^{1-c} P_{s_{i} \bar{u}, s_{i} \bar{v}}+q^{c} P_{\bar{u}, s_{i} \bar{v}}-\sum_{z \in \mathfrak{S}(n-1): i \in D_{L}(z)} \mu\left(z, s_{i} \bar{v}\right) P_{\bar{u}, z} \\
& =P_{\bar{u}, \bar{v}}(q)
\end{aligned}
$$

by Theorem 2.2.

We conclude this section with a simple characterization of the permutations that, if used as the second index, give rise to Kazhdan-Lusztig polynomials equal to 1 (see [9] for a proof). 
Let $\tau \in \mathfrak{S}(m)$ and $\sigma \in \mathfrak{S}(n)$ with $n \geq m$. We say that $\sigma$ avoids $\tau$ if there is no subsequence $1 \leq i_{1}<\cdots<i_{m} \leq n$ such that

$$
\sigma\left(i_{\tau(1)}\right)<\cdots<\sigma\left(i_{\tau(m)}\right) .
$$

Theorem 2.10 (Lakshmibai-Sandhya) Let $v \in \mathfrak{S}(n)$. Then

$$
P_{u, v}(q)=1 \forall u \leq v \Longleftrightarrow v \text { avoids both } 3412 \text { and } 4231 \text {. }
$$

\section{A reduction theorem}

Definition Let $u, v \in \mathfrak{S}(n)$. Then we set

$$
d(u, v):=u^{-1}(n)-v^{-1}(n)
$$

Note that by Theorem 2.1, if $u \leq v$ we have $d(u, v) \geq 0$.

We are going to reduce the calculation of $P_{u, v}(q)$ to a problem for Kazhdan-Lusztig polynomials for $\mathfrak{S}(n-1)$ when $d(u, v) \leq 3$. We have already seen that if $d(u, v)=0$ then $P_{u, v}(q)=P_{\bar{u}, \bar{v}}(q)$, so we may restrict our attention to the case $d(u, v)>0$.

The next results, for $d(u, v)=1$ or 2 , are a reformulation and a generalization of a theorem of F. Brenti and R. Simion (see [4, Theorem 3.1]).

Theorem 3.1 Let $u, v \in \mathfrak{S}(n)$ be such that $u \leq v$ and $i=v^{-1}(n)$. Then

1. If $d(u, v)=1$,

$$
P_{u, v}(q)=P_{\bar{u}, \bar{v}}(q)
$$

2. If $d(u, v)=2$,

$$
P_{u, v}(q)= \begin{cases}q^{1-c} P_{\bar{u} s_{i}, \bar{v}}(q)+q^{c} P_{\bar{u}, \bar{v}}(q), & \text { if } i+1 \notin D_{R}(v), \\ P_{\bar{u}, \bar{v}}(q), & \text { if } i+1 \in D_{R}(v),\end{cases}
$$

where $c=1$ if $i \in D_{R}(u)$ and $c=0$ otherwise.

Proof: Part 1 follows easily from Proposition 2.3 and Proposition 2.9.

The case $i+1 \in D_{R}(v)$ of part 2 is again a consequence of Proposition 2.3 and Proposition 2.9 so we may suppose $i+1 \notin D_{R}(v)$. By Theorem 2.5 and the first part of Theorem 3.1 we may write

$$
\begin{aligned}
P_{u, v}(q) & =q^{1-c} P_{u s_{i}, v s_{i}}(q)+q^{c} P_{u, v s_{i}}(q)-\sum_{z \in Z\left(u, v s_{i} ; i\right)} q^{\frac{\ell(z, v)}{2}} \mu\left(z, v s_{i}\right) P_{u, z}(q) \\
& =q^{1-c} P_{\bar{u} s_{i}, \bar{v}}(q)+q^{c} P_{\bar{u}, \bar{v}}(q)-\sum_{z \in Z\left(u, v s_{i} ; i\right)} q^{\frac{\ell(z, v)}{2}} \mu\left(z, v s_{i}\right) P_{u, z}(q)
\end{aligned}
$$


and hence it is enough to show that the sum appearing in this formula is actually zero in this case. Suppose $z \in Z_{1}\left(u, v s_{i} ; i\right)$. Then $i, i+1 \in D_{R}(z)$ and hence $z^{-1}(n)>i+2$ (since $\left.z \leq v s_{i}\right)$. But this implies that $u \not z z$ and hence $Z_{1}\left(u, v s_{i} ; i\right)=\emptyset$. It's not difficult to verify that also $Z_{2}\left(u, v s_{i} ; i\right)=\emptyset$ and the thesis follows.

Suppose now that $d(u, v)=3$ and again set $i=v^{-1}(n)$. To fix the ideas we write

$$
u:=\ldots u_{i} u_{i+1} u_{i+2} n \ldots
$$

and

$$
v:=\ldots n v_{i+1} v_{i+2} v_{i+3} \ldots
$$

If $v_{i+2}>v_{i+3}$ then, by Proposition 2.3, we may swap $u_{i+2}$ and $n$ in $u$ and hence we go back to the case $d(u, v)=2$. So, with no lack of generality, we may suppose $v_{i+2}<v_{i+3}$, i.e. $i+2 \notin D_{R}(v)$. We would like to use Theorem 2.5 taking $i$ as a right descent for $v$. The next result will allow us to simplify the sum in that formula in this case.

Lemma 3.2 Let $u, v \in \mathfrak{S}(n)$ be such that $u \leq v, d(u, v)=3, i=v^{-1}(n)$ and $i+2 \notin$ $D_{R}(v)$. Then the application $z \mapsto \bar{z}$ establishes a bijection between the following sets of permutations:

$$
\left\{\begin{array}{c}
z \in \mathfrak{S}(n) \text { such that } z \geq u \\
i \in D_{R}(z), \mu\left(z, v s_{i}\right) \neq 0 \\
\text { and } z \triangleleft v s_{i}
\end{array}\right\} \longleftrightarrow\left\{\begin{array}{c}
z \in \mathfrak{S}(n-1) \text { such that } z \geq \bar{u} \\
i, i+1 \in D_{R}(z) \\
\text { and } \mu(z, \bar{v}) \neq 0
\end{array}\right\}
$$

Moreover, if $z$ belongs to the set in the left-hand side, we have $\mu\left(z, v s_{i}\right)=\mu(\bar{z}, \bar{v})$, $\ell(z, v)=\ell(\bar{z}, \bar{v})+3$ and $P_{u, z}(q)=P_{\bar{u}, \bar{z}}(q)$.

Proof: Let $z$ be in the set in the left-hand side. The condition $z \geq u$ implies $z^{-1}(n) \leq i+3$ while the condition $z \leq v s_{i}$ implies $z^{-1}(n) \geq i+1$. But since $i, i+1 \in D_{R}(z)$ these conditions force $z^{-1}(n)=i+3$ which implies that, if the map is well defined, it is actually injective. Hence, locally, we have:

$$
z=\ldots e d c n \ldots
$$

with $e>d>c$ and

$$
v=\ldots n \times a b \ldots
$$

with $a<b$. Since $d\left(z, v s_{i}\right)=2$ we can use Theorem 3.1 to obtain

$$
\begin{aligned}
P_{z, v s_{i}}(q) & =P_{\ldots e c d n \ldots, \ldots x a n b \ldots}(q)+q P_{\ldots e d c n \ldots, \ldots x a n b \ldots}(q) \\
& =P_{\ldots e c d n \ldots, \ldots x a n b \ldots}(q)+q P_{\ldots e d n} \ldots \ldots, \ldots x a n \text { n } \ldots \ldots
\end{aligned}
$$


By Corollary 2.4 the first polynomial gives no contribution for $\mu\left(z, v s_{i}\right)$ and hence we may conclude that

$$
\begin{aligned}
\mu\left(z, v s_{i}\right) & =\mu\left(z s_{i+2}, v s_{i} s_{i+1}\right) \\
& =\mu\left(\overline{z s_{i+2}}, \overline{v s_{i} s_{i+1}}\right) \\
& =\mu(\bar{z}, \bar{v}),
\end{aligned}
$$

where we have used Propositions 2.3 and 2.9. It's a routine calculation that $z$ and $\bar{z}$ verify the other conditions of the statement and we are done.

We are now ready to state the main result of this section:

Theorem 3.3 Let $u, v \in \mathfrak{S}(n)$ be such that $u \leq v, d(u, v)=3, i=v^{-1}(n)$ and $i+2 \notin$ $D_{R}(v)$. Then

$$
\begin{aligned}
P_{u, v}(q)= & q^{2-c_{0,1}-c_{0,2}} P_{\bar{u} s_{i} s_{i+1}, \bar{v}}(q)+q^{1-c_{0,1}+c_{0,2}} P_{\bar{u} s_{i}, \bar{v}}(q)+q^{1+c_{0,1}-c_{1,2}} P_{\bar{u} s_{i+1}, \bar{v}}(q) \\
& +q^{c_{0,1}+c_{1,2}} P_{\bar{u}, \bar{v}}(q)-\sum_{\left\{z \in \mathfrak{S}(n-1): i, i+1 \in D_{R}(z)\right\}} q^{\frac{\ell(z, \bar{v})+3}{2}} \mu(z, \bar{v}) P_{\bar{u}, z}(q) \\
& -\varepsilon_{0} q P_{\bar{u}, \bar{v}}(q)-\varepsilon_{1} q P_{\bar{u}, \bar{v} s_{i+1}}(q)
\end{aligned}
$$

where

$$
\varepsilon_{j}:= \begin{cases}0, & \text { if } v_{i+1}<v_{i+j+2} \\ 1, & \text { otherwise }\end{cases}
$$

and

$$
c_{j, k}:= \begin{cases}0, & \text { if } u_{i+j}<u_{i+k}, \\ 1, & \text { otherwise. }\end{cases}
$$

Proof: The proof follows directly from Lemma 3.2 and Theorem 3.1. We only have to check that the contribution of $Z_{2}\left(u, v s_{i} ; i\right)$ is actually given by the last two polynomials and this verification is left to the reader (the only two candidates for $Z_{2}\left(u, v s_{i} ; i\right)$ are $v s_{i} s_{i+1}$ and $\left.v s_{i}(i+1, i+3) \ldots\right)$.

It should be mentioned that both Theorems 3.1 and 3.3 can also be stated in a "dual" version when $u, v \in \mathfrak{S}(n)$ satisfy $\tilde{d}(u, v):=v^{-1}(1)-u^{-1}(1) \leq 3$.

The next example will show us that, unfortunately, there can be many terms different from 0 in the sum appearing in Theorem 3.3.

Example 3.4 Let $n \geq 5$ and $v:=3 \ldots(n-2) n(n-1) 12$ (and hence $\bar{v}=3 \ldots(n-$ $2)(n-1) 12)$ and $u=e$. Then it is easy to check that for every $i \in[3, n-2],(1, i) \bar{v}$ gives rise to a non-zero summand in Theorem 3.3. 


\section{Main results}

The main goal of this section is to find an explicit formula for all polynomials $P_{u, v}(q)$, $u, v \in \mathfrak{S}(n)$, when $D_{R}(\bar{v}) \supseteq[2, n-2]$ and $u$ has some particular shape depending on that of $v$.

With this purpose we fix $x, y, n \in \mathbb{N}$ such that $x, y \in[2, n-1]$ and $x \neq y$. We denote by $\sigma_{0}$ the unique element $v$ of $\mathfrak{S}(n-1)$ such that $v(1)=x, v(n-1)=y$ and $[2, n-2] \subseteq D_{R}(v)$.

For any $i \in[n]$ we denote by $v_{i}$ the unique permutation of $\mathfrak{S}(n)$ satisfying the following two conditions:

$$
\begin{aligned}
& \overline{v_{i}}=\sigma_{0} \\
& v_{i}^{-1}(n)=i
\end{aligned}
$$

We also denote by $u_{i, a} \in \mathfrak{S}(n), \forall a, i \in \mathbb{N}, 1 \leq a \leq i \leq n$,

$$
u_{i, a}:=s_{a} s_{a+1} \ldots s_{i-1}
$$

so that, in particular, $u_{i, i}=e, \forall i \in[n]$.

For example, for $n=6, x=4$ and $y=2$ we have $v_{4}=453612, v_{5}=453162$ and $u_{4,3}=124356$.

We denote by $\mathcal{R}_{i, a}(q):=P_{u_{i, a}, v_{i}}(q)$. Note that $\mathcal{R}_{i, 1}(q)$ can be easily expressed as a linear combination of polynomials $\mathcal{R}_{i, a}$ with $a>1$ and suitable values of $x$ and $y$ by Proposition 2.3 and its "dual" version, so we only have to deal with the case $a>1$.

The following is the main result of this paper:

Theorem 4.1 Let $2 \leq a \leq i \leq n-3$. Then the polynomials $\mathcal{R}_{i, a}(q)$ satisfy the following relations: if $x>y$

$$
\mathcal{R}_{i, a}(q)=q \mathcal{R}_{i+1, a}(q)+\mathcal{R}_{i+1, i+1}(q)-q \mathcal{R}_{i+2, i+2}(q)-\delta_{i, n-y-1} q-\delta_{i, n-x} q^{x-y+1},
$$

and if $x<y$

$$
\mathcal{R}_{i, a}(q)=q \mathcal{R}_{i+1, a}(q)+\mathcal{R}_{i+1, i+1}(q)-q \mathcal{R}_{i+2, i+2}(q)-\delta_{i, n-y} q,
$$

where $\delta_{i, j}$ is the usual Kronecker symbol.

Proof: Case 1: $x>y$.

Let $i \in[2, n-3]$. By Theorem 2.5 and Proposition 2.3 we have:

$$
\begin{aligned}
\mathcal{R}_{i, a}(q) & =q P_{u_{i+1, a}, v_{i+1}}(q)+P_{u_{i+1, i+1}, v_{i+1}}(q)-\sum_{z \in Z\left(u_{i, a}, v_{i+1} ; i\right)} q^{\frac{\ell\left(z, v_{i}\right)}{2}} \mu\left(z, v_{i+1}\right) P_{u_{i, a}, z}(q) \\
& =q \mathcal{R}_{i+1, a}(q)+\mathcal{R}_{i+1, i+1}(q)-\sum_{z \in Z\left(u_{i, a}, v_{i+1} ; i\right)} q^{\frac{\ell\left(z, v_{i}\right)}{2}} \mu\left(z, v_{i+1}\right) P_{u_{i, a}, z}(q) .
\end{aligned}
$$


If $z \in Z_{1}\left(u_{i, a}, v_{i+1} ; i\right)$ we have [2,n-2] $\subseteq D_{R}(z)$ that forces $z^{-1}(n)=n$ (since $\left.z \leq v_{i+1}\right)$. Moreover the condition $[1, n-2] \backslash\{y-1, x\} \subseteq D_{L}(z)$ implies that $z(1) \in\{y-1, x, n-1\}$. But if $z(1)=n-1$ we have $z \not \leq v_{i+1}$ (unless $x=n-1$ but in this case it's okay to exclude $n-1$ since we still have $x$ ) and hence we have two possibilities for $z$, namely:

$$
\begin{aligned}
& \zeta_{1}=x n-1 \ldots \hat{x} \ldots 1 n \\
& \zeta_{2}=y-1 n-1 \ldots \widehat{y-1} \ldots 1 n .
\end{aligned}
$$

So we have to check whether $\mu\left(\zeta_{j}, v_{i+1}\right)=0$ or not for $j=1,2$.

Suppose $j=1$. If $i>n-y-1$ (i.e. $n$ appears after $y-1$ in the complete notation of $\left.v_{i+1}\right)$, it's easy to check that if we call $\tilde{\zeta}_{1}$ and $\tilde{v}_{i+1}$ the permutations obtained by suppressing the values $1,2 \ldots n-i-2$ (and rescaling) from $\zeta_{1}$ and $v_{i+1}$ respectively we have:

$$
\begin{aligned}
\mu\left(\zeta_{1}, v_{i+1}\right) & =\mu\left(\tilde{\zeta}_{1}, \tilde{v}_{i+1}\right) \\
& =\mu(x n-1 \ldots \hat{x} \ldots n-i-1 n, x n-1 \ldots \hat{x} \ldots \hat{y} \ldots n-i-1 n y) \\
& =0
\end{aligned}
$$

by Corollary 2.4 .

Similarly, if $i<n-y-1$, we have

$$
\begin{aligned}
\mu\left(\zeta_{1}, v_{i+1}\right) & =\mu(x n-1 \ldots \hat{x} \ldots y n, x n-1 \ldots y+1 y) \\
& =0 .
\end{aligned}
$$

Finally, if $i=n-y-1$ we have $\zeta_{1} \triangleleft v_{i+1}$ and hence it doesn't belong to $Z_{1}\left(u_{i, a}, v_{i+1} ; i\right)$ by definition.

Suppose now $j=2$. If $i \geq n-y-1$ then the same proof of the case $j=1$ implies $\mu\left(\zeta_{2}, v_{i+1}\right)=0$ and hence we may suppose that $i<n-y-1$. But in this case we have $\zeta_{2}^{-1}(h)=v_{i+1}^{-1}(h)$ for $h=1, \ldots, y-2$, so we can suppose $y=2$ with no lack of generality (substituting $x$ with $x-y+2$ and $n$ by $n-y+2$ ). So we have

$$
v_{i+1}=x n-1 \ldots n \ldots 312
$$

where $n$ appears in position $i+1$ and

$$
\zeta_{2}=1 n-1 \ldots 2 n
$$

By Proposition 2.7 we can compute $\mu\left(w_{0} v_{i+1}, w_{0} \zeta_{2}\right)$ instead of $\mu\left(\zeta_{2}, v_{i+1}\right)$.

If $n-x-i+1 \geq 0$ we have

$$
w_{0} v_{i+1}=s_{1} \ldots s_{i-1} s_{n-1} s_{n-x} \ldots s_{1}
$$

and

$$
w_{0} \zeta_{2}=s_{1} s_{2} \ldots s_{n-1} s_{n-2} \ldots s_{1} .
$$


Hence, by Theorem 2.6, we have that $\mu\left(w_{0} v_{i+1}, w_{0} \zeta_{2}\right) \neq 0$ if and only if $i=n-x$ and in this case $\mu\left(w_{0} v_{i+1}, w_{0} \zeta_{2}\right)=1$.

If $n-x-i+1<0$ we have

$$
w_{0} v_{i+1}=s_{1} \ldots s_{i} s_{n-1} s_{n-x-1} \ldots s_{1}
$$

so, by Theorem 2.6, we have $\mu\left(w_{0} v_{i+1}, w_{0} \zeta_{2}\right)=0$.

Now it is an easy exercise to verify that $\ell\left(\zeta_{2}, v_{n-x}\right)=2(x-y+1)$ and that $P_{u_{n-x, a}, \zeta_{2}}(q)$ $=1$.

Finally we leave to the reader to verify that

$$
Z_{2}\left(u_{i, a}, v_{i+1} ; i\right)= \begin{cases}\left\{v_{i+2}\right\}, & \text { if } i \neq n-y-1 \\ \left\{v_{i+2}, x n-1 \ldots \hat{x} \ldots 1 n\right\}, & \text { if } i=n-y-1\end{cases}
$$

and the proof is complete.

Case 2: $x<y$. With an argument similar to that of Case 1 (and actually easier) we can prove that $Z_{1}\left(u_{i, a}, v_{i+1} ; i\right)=\emptyset$.

As before we have

$$
Z_{2}\left(u_{i, a}, v_{i+1} ; i\right)= \begin{cases}\left\{v_{i+2}\right\}, & \text { if } i \neq n-y, \\ \left\{v_{i+2}, x n-1 \ldots \hat{x} \ldots 1 n\right\}, & \text { if } i=n-y,\end{cases}
$$

and we are done.

Theorems 3.1 and 4.1 can be used to find explicit formulae for the polynomials $\mathcal{R}_{i, a}(q)$. Recall that we have defined, for all $n \in \mathbb{Z},[n]_{q}=\sum_{j=0}^{n-1} q^{j}$. We first need the following observation about this function that we state as a lemma for future reference.

Lemma 4.2 Let $n \in \mathbb{Z}$. Then

$$
(q+1)[n]_{q}-q[n-1]_{q}=[n+1]_{q}-\delta_{0, n} .
$$

Corollary 4.3 Let $n, x, y \in \mathbb{N}$ be such that $2 \leq y<x \leq n-1$. Denote by

$$
\begin{aligned}
H_{i}(q):= & q[n-y-i]_{q}+q^{n-y-1}[x-i]_{q}+q^{x-y+1}[n-x+1-i]_{q} \\
& +q^{n-y}[y-1-i]_{q} .
\end{aligned}
$$

Then, for $2 \leq a \leq i$, we have:

$$
\mathcal{R}_{i, a}(q)= \begin{cases}\left(1+q^{x-y}\right)[n-1-i]_{q}-H_{i}(q), & \text { if } a \in[2, y-1], \\ {[n-i]_{q}+q^{x-y}[n-1-i]_{q}-H_{i}(q),} & \text { if } a \in[y, x], \\ \left(1+q^{x-y}\right)[n-i]_{q}-H_{i}(q), & \text { if } a \in[x+1, n-1] .\end{cases}
$$


Proof: We proceed by a double induction on $n$ and $n-i$. If $n=4$ the statement is an easy verification. So suppose that the statement is true for $n-1$. If $i=n-2, n-1, n$ then, by Theorem 3.1, $\mathcal{R}_{i, a}(q)$ can be easily reduced to the $n-1$ case and hence it is a simple verification. So suppose $i<n-2$. By Lemma 4.2 it follows directly that

$$
\begin{aligned}
(q+1) H_{i+1}-q H_{i+2}= & H_{i}-\delta_{0, n-y-i-1} q-\delta_{0, x-i-1} q^{n-y-1} \\
& -\delta_{0, n-x-i} q^{x-y+1}-\delta_{0, y-i-2} q^{n-y}
\end{aligned}
$$

Denote by

$$
\varepsilon_{j}:=\left\{\begin{array}{ll}
1, & \text { if } j<y, \\
0, & \text { otherwise }
\end{array} \quad \text { and } \quad \eta_{j}:= \begin{cases}1, & \text { if } j \leq x, \\
0, & \text { otherwise. }\end{cases}\right.
$$

Observe that we have $\left[n-i-1-\varepsilon_{i+1}\right]_{q}-q\left[n-i-2-\varepsilon_{i+2}\right]_{q}=\left(1-\delta_{i+1, y-1} q^{n-i-2}\right)$ and a similar equation where $\varepsilon$ is substituted by $\eta$. We have, by Theorem 4.1 and (4.1),

$$
\begin{aligned}
\mathcal{R}_{i, a}(q)= & q \mathcal{R}_{i+1, a}(q)+\mathcal{R}_{i+1, i+1}(q)-q \mathcal{R}_{i+2, i+2}(q)-\delta_{i, n-y-1} q-\delta_{i, n-x} q^{x-y+1} \\
= & q\left[n-i-1-\varepsilon_{a}\right]_{q}+q q^{x-y}\left[n-i-1-\eta_{a}\right]_{q}+\left[n-i-1-\varepsilon_{i+1}\right]_{q} \\
& +q^{x-y}\left[n-i-1-\eta_{i+1}\right]-H_{i}-q\left[n-i-2-\varepsilon_{i+2}\right]_{q} \\
& -q q^{x-y}\left[n-i-2-\eta_{i+2}\right]_{q}+\delta_{0, x-i-1} q^{n-y-1}+\delta_{0, y-i-2} q^{n-y} \\
= & q\left[n-i-1-\varepsilon_{a}\right]_{q}+q q^{x-y}\left[n-i-1-\eta_{a}\right]_{q}+\left(1-\delta_{i+1, y-1} q^{n-i-2}\right) \\
& +q^{x-y}\left(1-\delta_{i+1, x} q^{n-i-2}\right)-H_{i}+\delta_{0, x-i-1} q^{n-y-1}+\delta_{0, y-i-2} q^{n-y} \\
= & {\left[n-i-\varepsilon_{a}\right]_{q}+q^{x-y}\left[n-i-\eta_{a}\right]_{q}-H_{i} }
\end{aligned}
$$

and the proof is complete.

Corollary 4.4 Let $n, x, y \in \mathbb{N}$ be such that $2 \leq x<y \leq n-1$. Denote by

$$
K_{i}(q):=q[n-y+1-i]_{q}+q^{n-y}[y-1-i]_{q} .
$$

Then, for $2 \leq a \leq i$, we have:

$$
\mathcal{R}_{i, a}(q)= \begin{cases}{[n-1-i]_{q}-K_{i}(q),} & \text { if } a \in[2, y-1], \\ {[n-i]_{q}-K_{i}(q),} & \text { if } a \in[y, n-1] .\end{cases}
$$

Proof: We proceed, as in the proof of Corollary 4.3, by a double induction on $n$ and $n-i$. Again the case $n=4$ is an easy verification and the cases $i=n-2, n-1, n$ follow directly from Theorem 3.1. So suppose $i<n-2$. By Lemma 4.2 we have

$$
(q+1) K_{i+1}-q K_{i+2}=K_{i}-\delta_{0, n-y-i} q-\delta_{0, y-i-2} q^{n-y} .
$$


Then, by Theorem 4.1, we have that

$$
\begin{aligned}
\mathcal{R}_{i, a}(q)= & q \mathcal{R}_{i+1, a}(q)+\mathcal{R}_{i+1, i+1}(q)-q \mathcal{R}_{i+2, i+2}(q)-\delta_{i, n-y} q \\
= & q\left[n-i-1-\varepsilon_{a}\right]_{q}+\left[n-i-1-\varepsilon_{i+1}\right]_{q}-q\left[n-i-2-\varepsilon_{i+2}\right]_{q} \\
& -K_{i}(q)+\delta_{0, y-i-2} q^{n-y} \\
= & q\left[n-i-1-\varepsilon_{a}\right]_{q}+\left(1-\delta_{i+1, y-1} q^{n-y}\right)-K_{i}(q)+\delta_{0, y-i-2} q^{n-y} \\
= & {\left[n-i-\varepsilon_{a}\right]_{q}-K_{i}(q) }
\end{aligned}
$$

where, for $j \in[n]$,

$$
\varepsilon_{j}:=\left\{\begin{array}{ll}
1, & \text { if } j<y, \\
0, & \text { otherwise. }
\end{array} \quad \chi(j<y)\right.
$$

Note that in this case $\mathcal{R}_{i, a}(q)$ doesn't depend on $x$.

Remark By the explicit formulae appearing in Corollaries 4.3 and 4.4, it is easy to see that all the polynomials $\mathcal{R}_{i, a}(q)$ have nonnegative coefficients.

Corollaries 4.4 and 4.3 give us an elementary proof of the following theorem of Shapiro, Shapiro and Vainshtein [13, Theorem 1], which was originally proved in a geometric way:

Corollary 4.5 Let $n \geq 3$ and $u, v \in \mathfrak{S}(n), u \leq v$, be such that $[2, n-2] \subseteq D_{R}(v)$. Then

$$
P_{u, v}(q)= \begin{cases}1, & \text { if } v_{1}<v_{n}, \text { or } v_{n} \leq u_{1}, \text { or } v_{1} \geq u_{n}, \\ 1+q^{v_{1}-v_{n}}, & \text { otherwise }\end{cases}
$$

Proof: We proceed by induction on $n$. If $n=3$ the statement is trivial, so suppose $n>3$. By Proposition 2.3 we can suppose that $u_{2}<u_{3}<\cdots<u_{n-1}$. If $u \neq e$ or $\left\{v_{1}, v_{n}\right\} \cap\{1, n\} \neq \emptyset$ we have that

$$
P_{u, v}(q)=P_{\bar{u}, \bar{v}}(q) \quad \text { or } \quad P_{u, v}(q)=P_{\underline{u}, \underline{v}}(q)
$$

by Propositions 2.3 and 2.9 and the thesis follows by induction. If $u=e$ and $\left\{v_{1}, v_{n}\right\} \cap$ $\{1, n\}=\varnothing$

$$
P_{e, v}(q)=\mathcal{R}_{2,2}(q)= \begin{cases}1+q^{v_{1}-v_{n}}, & \text { if } v_{1}>v_{n} \\ 1, & \text { if } v_{1}<v_{n}\end{cases}
$$

and we are done.

The following two results have been conjectured by Brenti and Simion [4, Conjectures 4.2 and 4.3]: 
Corollary 4.6 Let $n \geq 6$. Then

$$
P_{e, n-2 n-1 n n-3 \ldots 4213}(q)=1+2 q^{n-5}+q^{n-4} \text {. }
$$

Proof: Set $x=n-2$ and $y=3$. Then

$$
\begin{aligned}
P_{e, n-2 n-1 n n-3 \ldots 4213}(q) & =\mathcal{R}_{3,3}(q) \\
& =[n-3]_{q}+q^{n-5}[n-4]_{q}-q[n-6]_{q}-q^{n-4}[n-5]_{q} \\
& =1+2 q^{n-5}+q^{n-4} .
\end{aligned}
$$

Corollary 4.7 Let $n \geq 6$. Then

$$
P_{e, n-2 n-1 n n-3 \ldots 312}(q)=1+2 q^{n-4} \text {. }
$$

Proof: $\quad$ Set $x=n-2$ and $y=2$. Then

$$
\begin{aligned}
P_{e, n-2 n-1 n n-3 \ldots 312}(q) & =\mathcal{R}_{3,3}(q) \\
& =[n-3]_{q}+q^{n-4}[n-4]_{q}-q[n-5]_{q}-q^{n-3}[n-5]_{q} \\
& =1+2 q^{n-4} .
\end{aligned}
$$

The conjectures of Brenti and Simion suggest, more generally, the problem of computing $P_{e, v}$ when the first and the last three entries of $v$ are respectively any permutation of the sets $\{n-2, n-1, n\}$ and $\{1,2,3\}$, and $[4, n-4] \subseteq D_{R}(v)$.

With this purpose we let $n \in \mathbb{N}, n \geq 6$, and $\mathfrak{S}(3)$ act at the same time on $\{1,2,3\}$ in the usual way and on $\{n-2, n-1, n\}$ in the natural way identifying $n-2, n-1$ and $n$ with 1,2 and 3 respectively.

Definition $\forall(\sigma, \tau) \in \mathfrak{S}(3) \times \mathfrak{S}(3)$ we denote by $D_{\sigma, \tau}(q)$ the following Kazhdan-Lusztig polynomial:

$$
D_{\sigma, \tau}(q):=P_{e, \sigma(n-2) \sigma(n-1) \sigma(n) n-3 \ldots 4 \tau(1) \tau(2) \tau(3)}(q) .
$$

We conclude by showing that (unless $\sigma=\tau=e$ ) all these polynomials admit simple explicit formulas:

Theorem 4.8 $\forall n \geq 6$ the following formulae hold:

(1) $D_{123,321}(q)=D_{321,123}(q)=1$,

(2) $D_{132,321}(q)=D_{321,132}(q)=D_{321,213}(q)=D_{213,321}(q)=1$,

(3) $D_{231,321}(q)=D_{321,312}(q)=1$,

(4) $D_{321,321}(q)=1$,

(5) $D_{312,321}(q)=D_{321,231}(q)=1+q$,

(6) $D_{231,312}(q)=1+q^{n-3}$, 
(7) $D_{213,312}(q)=D_{231,132}(q)=D_{231,213}(q)=D_{132,312}(q)=1+q^{n-4}$,

(8) $D_{132,213}(q)=D_{213,132}(q)=1+q^{n-5}$,

(9) $D_{132,132}(q)=D_{213,213}(q)=1+q^{n-5}(1+q)$,

(10) $D_{123,312}(q)=D_{231,123}(q)=1+2 q^{n-4}$,

(11) $D_{123,132}(q)=D_{132,123}(q)=D_{213,123}(q)=D_{123,213}(q)=1+q^{n-5}(2+q)$,

(12) $D_{123,231}(q)=D_{312,123}(q)=(1+q)\left(1+2 q^{n-5}\right)$,

(13) $D_{231,231}(q)=D_{312,312}(q)=1+q+q^{n-4}$,

(14) $D_{132,231}(q)=D_{312,132}(q)=D_{312,213}(q)=D_{213,231}(q)=(1+q)\left(1+q^{n-5}\right)$,

(15) $D_{312,231}(q)=(1+q)^{2}\left(1+q^{n-5}\right)$.

Proof: All the equalities among the $D_{\sigma, \tau}(q)$ 's in each row of Theorem 4.8 are due to Theorem 2.8 while Eqs. (1)-(4) follows directly from Theorem 2.10. Equations (6)-(11) are particular cases of the explicit formulae appearing in Corollary 4.3.

We sketch the proofs of the other cases leaving the details to the reader:

(5)

$$
\begin{aligned}
P_{e, n n-2 n-1 n-3 \ldots 1}(q) & =q P_{S_{1}, n-2 n n-1 n-3 \ldots 1}(q)+P_{e, n-2 n n-1 n-3 \ldots 1}(q) \\
& =q+1,
\end{aligned}
$$

by Theorem 2.10 .

(12) We would like to compute $P_{e, n-2 n-1 n n-3 \ldots .4231}(q)$ using Theorem 2.5 taking $i=$ $n-1$. It's not difficult to check that $Z_{1}(e, n-2 n-1 n n-3 \ldots 4213 ; n-1)=\{21 n \ldots 3\}$. But

$$
\begin{aligned}
& \mu(21 n \ldots 3, n-2 n-1 n n-3 \ldots 4213) \\
& \quad=\mu(21 n-1 \ldots 3, n-2 n-1 n-3 \ldots 4213) \\
& \quad=0
\end{aligned}
$$

by Corollary 2.4, since $2 \in D_{R}(n-2 n-1 n-3 \ldots 4213) \backslash D_{R}(21 n \ldots 3, n-2 n-1 n n-$ $3 \ldots 4213)$. It's easy to check that $Z_{2}(e, n-2 n-1 n n-3 \ldots 4213 ; n-1)=\emptyset$, so we may write:

$$
\begin{aligned}
P_{e, n-2 n-1 n n-3 \ldots 4231}(q) & =q P_{s_{n-1}, n-2 n-1 n n-3 \ldots 4213}(q)+P_{e, n-2 n-1 n n-3 \ldots 4213}(q) \\
& =q P_{e, n-2 n-1 n-3 \ldots 4231}(q)+P_{e, n-2 n-1 n n-3 \ldots 4213}(q) \\
& =q\left(1+q^{n-5}\right)+1+2 q^{n-5}+q^{n-4} \\
& =(1+q)\left(1+2 q^{n-5}\right),
\end{aligned}
$$

by Corollary 4.3 .

(13) Using Theorem 2.5 it's easy to see that

$$
\begin{aligned}
P_{e, n-1 n n-2 \ldots 4231}(q) & =q P_{s_{n-1}, n-1 n n-2 \ldots .4213}(q)+P_{e, n-1 n n-2 \ldots 4213}(q) \\
& =q P_{e, n-1 n-2 \ldots . .4213}(q)+P_{e, n-1 n n-2 \ldots 4213}(q) \\
& =1+q+q^{n-4}
\end{aligned}
$$


by Corollary 4.3 .

(14) Always by Theorem 2.5 we have:

$$
\begin{aligned}
P_{e, n n-2 n-1 n-3 \ldots 4213}(q) & =q P_{s_{1}, n-2 n n-1 n-3 \ldots 4213}(q)+P_{e, n-2 n n-1 n-3 \ldots 4213}(q) \\
& =q\left(1+q^{n-5}\right)+1+q^{n-5} \\
& =(1+q)\left(1+q^{n-5}\right),
\end{aligned}
$$

by Corollary 4.3 .

(15) In this case we have $Z_{1}(e, n n-2 n-1 n-3 \ldots 4213 ; n-1)=\{21 n \ldots 3\}$.

In fact, if $z \in Z_{1}(e, n n-2 n-1 n-3 \ldots 4213 ; n-1)$ we have $\{1\} \cup[3, n-1] \subseteq D_{R}(z)$ forcing $z^{-1}(1)=2$ (since $z \leq n n-2 n-1 n-3 \ldots 4213$ ). Moreover the condition $1 \in D_{L}(z)$ forces $z=21 n \ldots 3$.

Now, by Theorem 3.1, we have

$$
\begin{aligned}
P_{21 n \ldots 3, n n-2 n-1 n-3 \ldots 4213}(q)= & P_{12 n \ldots 3, n-2 n n-1 n-3 \ldots 4213}(q) \\
& +q P_{21 n \ldots 3, n-2 n n-1 n-3 \ldots 4213}(q) .
\end{aligned}
$$

This implies that

$$
\begin{aligned}
\mu(z, n n-2 n-1 n-3 \ldots 4213) & =\mu(21 n-1 \ldots 3, n-2 n-1 n-3 \ldots 4213) \\
& =0
\end{aligned}
$$

by Corollary 2.4, since $2 \in D_{R}(n-2 n-1 n-3 \ldots 4213) \backslash D_{R}(21 n-1 \ldots 3)$.

So we have, by Theorem 2.5 and repeated use of Proposition 2.3,

$$
\begin{aligned}
P_{e, n n-2 n-1 n-3 \ldots 4231}(q) & =q P_{s_{n-1}, n n-2 n-1 n-3 \ldots 4213}(q)+P_{e, n n-2 n-1 n-3 \ldots 4213}(q) \\
& =q P_{12 n 3 \ldots n-1, n n-2 n-1 n-3 \ldots 4213}(q)+(1+q)\left(1+q^{n-5}\right),
\end{aligned}
$$

by (14). So, by Theorem 3.1 and Proposition 2.9 we may conclude that

$$
\begin{aligned}
P_{e, n n-2 n-1 n-3 \ldots 4231}(q)= & q^{2} P_{21 n 3 \ldots n-1, n-2 n n-1 n-3 \ldots 4213}(q) \\
& +q P_{12 n 3 \ldots n-1, n-2 n n-1 n-3 \ldots 4213}(q)+(1+q)\left(1+q^{n-5}\right) \\
= & q^{2} P_{23 \ldots n-1, n-2 n-1 n-3 \ldots 423}(q)+q\left(1+q^{n-5}\right) \\
& +(1+q)\left(1+q^{n-5}\right) \\
= & q^{2}\left(1+q^{n-5}\right)+(1+2 q)\left(1+q^{n-5}\right) \\
= & (1+q)^{2}\left(1+q^{n-5}\right),
\end{aligned}
$$

by (7).

Remark 4.9 The only missing case from Theorem 4.8 is $D_{123,123}(q)$. This has turned out to be much more difficult than the others and will be treated apart in a joint work of the author and M. Marietti. 


\section{References}

1. B.D. Boe, "Kazhdan-Lusztig polynomials for hermitian symmetric spaces," Trans. Amer. Math. Soc. 309 (1988), 279-294.

2. F. Brenti, "A combinatorial formula for Kazhdan-Lusztig polynomials," Invent. Math. 118 (1994), 371-394.

3. F. Brenti, "Combinatorial properties of the Kazhdan-Lusztig $R$-polynomials for $S_{n}$," Adv. Math. 126 (1997), 21-51.

4. F. Brenti and R. Simion, "Explicit formulae for some Kazhdan-Lusztig polynomials," J. Alg. Comb. 11 (2000), 187-196.

5. C. Ehresmann, "Sur la topologie de certains espaces homogènes," Ann. Math. 35 (1934), 396-443.

6. J.E. Humphreys, Reflection Groups and Coxeter Groups, Cambridge Univ. Press, Cambridge, 1990.

7. D. Kazhdan and G. Lusztig, "Representations of Coxeter groups and Hecke algebras," Invent. Math. 53 (1979), 165-184.

8. D. Kazhdan and G. Lusztig, "Schubert varieties and Poincaré duality, Geometry of the Laplace operator," Proc. Symp. Pure Math. 34, Amer.Math. Soc., Providence, RI, 1980, 185-203.

9. V. Lakshmibai and B. Sandhya, "Criterion for smoothness of Schubert varieties in $S l(n) / B$," Proc. Indian Acad. Sci. Math. Sci. 100 (1990), 45-52.

10. A. Lascoux and M.P. Schützenberger, "Polynômes de Kazhdan-Lusztig pour les grassmanniennes," Young tableaux and Schur functions in algebra and geometry, Astérisque 87/88 (1981), 249-266.

11. M. Marietti, "Kazhdan-Lusztig polynomials for Boolean elements in linear Coxeter systems," preprint.

12. P. Polo, "Construction of arbitrary Kazhdan-Lusztig polynomials in symmetric groups," Representation Theory 3 (1999), 90-104.

13. B. Shapiro, M. Shapiro and A. Vainshtein, "Kazhdan-Lusztig polynomials for certain varieties of incomplete flags," Discr. Math. 180 (1998), 345-355. 